\title{
Special Issue on Artificial Intelligence in Agriculture
}

\author{
Andreas Dengel
}

Published online: 6 September 2013

(C) Springer-Verlag Berlin Heidelberg 2013

Agriculture and AI-intuitively, these domains seem to denote two separate worlds. Agriculture processes the soil and cares about food production and elementary supply-this is really down-to-earth! AI, in contrast, is deeply interwoven with computerized systems, complex interactions, modeling and reasoning approaches, and in public perception still suffers from flair of science fiction. So why do we dedicate the present special issue to this combination?

Modern Agriculture faces tremendous challenges. Today, the agricultural sector has grown into a highly competitive and globalized industry, where farmers and other actors have to consider local climatic and geographic aspects as well as global ecological and political factors in order to guarantee economical survival and sustainable production. Feeding a growing world population asks for continuous increases in food production, but arable land remains a limited resource. New requests for bio energy or changing diet preferences put additional strains on agricultural production, while settlement and transport consume increasing shares of land. Expected and observable changes in global climate, shifting rainfall patterns, global warming, droughts, or the increasing frequency and duration of extreme weather events endanger traditional production areas and bring new risks and uncertainties for global harvest yields. To cope with these challenges, Agriculture requires a continuous and sustainable increase in productivity and efficiency on all levels of agricultural production, while resources like water, energy, fertilizers etc. need to be used carefully and efficiently in order to protect and sustain the environment and the soil quality of the arable land. The complexity of the challenge is

A. Dengel $(\varangle)$

DFKI GmbH, Trippstadter Straße 122, 67663 Kaiserslautern, Germany

e-mail: Andreas.Dengel@dfki.de increased by other short-term events which are difficult to predict, such as epidemics, financial crisis, or price volatility for agricultural raw materials and products.

Consequently, Agriculture needs help in handling the complexity, uncertainty and fuzziness inherent in this domain, and it requires new solutions for all aspects of agricultural production-from better and predictable crop planning, to precision farming, optimized resource application, support of efficient and collaborative processes using modern technology, fully or partially autonomous solutions for tedious work, up to the sustained long-term development of useful knowledge resources.

The basis for computerized answers to such challenges in Agriculture has been realized in the recent decades: GPS (providing precise location data and offering the basis of all kinds of location-specific support) and mobile communication (allowing for the quick exchange of data between participants even in the field) are crucial and well-accepted breakthrough technologies. Making sense from the data that become available now, and using the resulting knowledge for process and operation improvement on all levels, brings into play AI and their modeling and reasoning capabilities.

From the AI point of view, Agriculture offers a vast application area for all kinds of AI core technologies: Mobile, autonomous agents operating in uncontrolled environments, stand-alone or in collaborative settings, allow to investigate, test and exploit technologies from robotics, computer vision, sensing, and environment interaction. Integrating multiple partners and their heterogeneous information sources leads to application of semantic technologies. The complexity of the agricultural production asks for progress in modeling capabilities, handling of uncertainty, and in the algorithmic and usability aspects of location- and context-specific decision support. The growing interest in reliable predictions as a basis for planning and control of agricultural activi- 
ties requires the interdisciplinary cooperation with domain experts e.g. from agricultural research. Modern agricultural machines shall use self-configuring components and shall be able to collaborate and exhibit aspects of self-organization and swarm intelligence.

After a intensive reviewing, we selected four technical contributions, three reports, and two doctoral theses summaries, which are complemented by an interview with Joachim Keizer from FAO on THE ROLE OF LINKED DATA IN AGRICULTURE.

All technical papers in this Special Issue give a first view on this challenging interplay between AI and Agriculture. Taking profit from state-of-the-art sensing and actuator technologies the contribution on DATA MINING AND PATTERN RECOGNITION IN AGRICULTURE addresses challenges and potentials of appropriate methods in Agriculture. Motivated by the need for increased resource efficiency, the paper on ROBOTS FOR FIELD OPERATION WITH COMPREHENSIVE MULTILAYER CONTROL summarizes work on the development of autonomous agricultural machines. A contribution to better understanding between multiple cooperating actors is proposed in a submission on ONTOLOGY-BASED MOBILE COMMUNICATION. Optimizing the operation of a harvesting logistics chain, consisting of multiple cooperating vehicles in the field, will profit from the application of dynamic route planning algorithms, as presented in a paper on SPATIAL-TEMPORAL CONSTAINT PLANNING.

While the report on the iGREEN project spans from support for sharing and exchange among agricultural operators to decision support and application control, the report on TOWARDS SUPPORTING MOBILE BUSINESS PROCESSES focuses on the uncertainty encountered in the nondeterministic agricultural environment and the application of agent technology to cope with that. Innovative ways for agricultural agents to see and perceive their environment are described in DETECTION OF FIELD STRUCTURES, which combines laser scanners and computer vision with sophisticated modeling capabilities to enable the intended structure recognition. In addition

Progress results in successful and interesting doctoral dissertation work: In order to enable self-organized sensor integration in modular machines, BIO-INSPIRED SENSOR DATA MANAGEMENT took inspiration from ant colonies and similar observations. MECHATRONIC SYSTEMS investigates the adaptation of the operating parameters of a modern agricultural machine to the current context and task details in the field.

The overview given in this special issue is far from complete. On one hand, we only present work in the context of agricultural plant production. Cattle breeding, e.g., offer interesting examples for applied robotics or advanced sensing and crowd monitoring - none of this is covered in this issue. On the other hand, the articles are very much focused on individual actors or machinery and their interaction and collaboration within the initial production. Multi-stage processes, involving finishing and sales organizations (think about mills, bakery, and retail shops) or sustained operation over the year (see e.g. the operation of a biogas plant) are not covered. There is also no contribution on the handling of extra-agricultural issues like e.g. the financial markets.

Nevertheless, the collection of articles shows that Agriculture is a promising application field for AI technology, and in turn AI has a rich variety of important contributions to offer to cope with the pressing challenges faced by Agriculture. We are looking forward to further interesting work to our mutual benefit!

\section{Content}

\subsection{Technical Contributions}

- Data Mining and Pattern Recognition in Agriculture Christian Bauckhage, Kerstin Kersting

- Robots for Field Operations with Comprehensive Multilayer Control

Hans.W. Griepentrog, Claes L. Dühring Jäger, Dimitrios Paraforos

- Ontology-Based Mobile Communication in Agriculture Malte Kiesel, Gunnar A. Grimnes, Ansgar Bernardi

- Spatio-temporally Constrained Planning for Cooperative Vehicles in a Harvesting Scenario

Stephan Scheuren, Stefan Stiene, Ronny Hartando, Joachim Hertzberg, Max Reinecke

\subsection{Research Projects}

- iGreen-Intelligent technologies for public-private knowledge management in agriculture

Ansgar Bernardi

- Detection of Field Structures for Agricultural Vehicle Guidance

Patrick Fleischmann, Tobias Föhst, Karsten Berns

- Towards supporting mobile business processes in nondeterministic agricultural environments by using agentbased technologies

Thilo Steckel, Thomas Kersting, Willi Nüßer

\subsection{Interview}

- The Role of Linked Data in Agriculture (with J. Keizer)

\subsection{Doctoral and Postdoctoral Dissertations}

- Bio-Inspired Sensor Data Management for Modular Agricultural Machines

Sebastian Blank

- Mechatronic system for mechanical weed control of the intra-row area in row crops

Zoltan Gobor 


\section{Service}

\subsection{Journals}

Journals focusing on AI/Computer Science and Agriculture include:

- Control an Intelligent Systems Journal http://www.actapress.com/Content_of_Journal.aspx? JournalID $=115$

- International Journal of Engineering Sciences and Emerging Technologies http://www.ijeset.com/

- International Journal of Robotics and Automation (IJRA) http://actapress.com/Content_of_Journal.aspx?JournalID $=119 \#$ \#info- 2

\subsection{Books}

There is a collection of books that may help interesting readers to get a broader impression of how knowledge technologies may help in agriculture. The below mentioned books represent an except of sources.

- Panigrahi, S. \& Ting K.C. (1998) Artificial Intelligence for Biology and Agriculture, Kluwer Academic Press).

- Maurer L. \& Tochtermann, K. (2009) Sustainability for Agriculture Through Knowledge Management, Information and Communication Technologies for Biodiversity \& Agriculture, Shaker Publ. (2009).

- Mukhopadhyay S.C. (2012) Smart Sensing Technology for Agriculture and Environmental Monitoring. Vol. 146, Springer Berlin Heidelberg.

- Sicilia M.-A. \& Lytras, M.D. (2009) Metadata and Semantics, Springer Publ.

- Li, D. \& Chen, Y. (1012) Computer and Computing Technologies in Agriculture VI, IFIP Advances in Information and Communication Technology, Vol. 393, Springer Publ.

- German, L., Ramisch, J.J. \& Verma R. (2010) Beyond the Biophysical, Knowledge, Culture, and Power in Agriculture and Natural Resource Management, Springer Publ.

- German, L., Ramisch, J.J. \& Verma R. (2010) Beyond the Biophysical, Knowledge, Culture, and Power in Agriculture and Natural Resource Management, Springer Publ.

\subsection{Conferences and Workshops}

There are no conferences and workshops yet that are specifically related to AI and Agriculture. However, the following forums regularly present respective papers.
- International Conference on Intelligent Agriculture http://www.icoia.org/

- IFAC Bio-Robotics Conference http://www.myray.com.my/v1/index.php/news-event/182013-ifac-bio-robotics-conference

- IEEE Biosignal \& Biorobotics Conference http://www.brc2013.org/

- International Conference on Sensing Technology http://seat.massey.ac.nz/conferences/icst2013/

- DMA Workshop on Data Mining in Agriculture http://www.wikicfp.com/cfp/servlet/event.showcfp? eventid=21516

- Agritechnica: Bi-Annual Fair \& Exhibition www.agritechnica.de

- International Conference on Agricultural Engineering (AgEng) http://cigr.ageng2012.org/

- European Federation for Information Technology in Agriculture, Food and Environment (EFITA) http://www.efita.net

\subsection{Organizations}

Some national and international organizations focus on the standardization and metadata generation such as:

- Food and Agricultural Organization oft the United Nations (FAO) http://www.fao.org/home/en/

- Kuratorium für Technik und Bauwesen in der Landwirtschaft e.V., Darmstadt (KTBL) http://www.ktbl.de/

\subsection{Software}

There is a large metadata collection, which may be found at:

- Vocabularies \& Metadata AIMS: Agriculture Information Management Standards of the FAO - Food and Agriculture Organization of the United Nations http://www.fao.org/

Acknowledgements I like to thank all authors of the various papers for their valuable contributions making this special issue possible. I further like to thank all reviewers for their thorough and timely reviewing of the submissions. I am also grateful to Ansgar Bernardi, Klaus-Dieter Althoff, and Ubbo Visser, who gently supported me during the whole process of preparation. 